This is the author's version of a work that was accepted for publication in Materials Chemistry and Physics. Changes resulting from the publishing process, such as peer review, editing, corrections, structural formatting and other quality control mechanisms may not be reflected in this document. Changes may have been made to this work since it was submitted for publication. A definitive version was subsequently published in Materials Chemistry and Physics, Volume 147, Issues 1-2, 15 September 2014, Pages 311-318. http://doi.org/10.1016/j.matchemphys.2014.05.006 


\title{
Structural dependence of threshold displacement energies in rutile, anatase and brookite $\mathrm{TiO}_{2}$
}

\author{
M. Robinson ${ }^{\mathrm{a}, *}$, N A Marks ${ }^{\mathrm{a}}$, G R Lumpkin ${ }^{\mathrm{b}}$ \\ ${ }^{a}$ Nanochemistry Research Institute, Curtin University, GPO Box U1987, Perth WA 6845, \\ Australia \\ ${ }^{b}$ Australian Nuclear Science and Technology Organisation, Locked Bag 2001, Kirrawee DC \\ NSW 2232, Australia
}

\begin{abstract}
Systematic molecular dynamics simulations of low energy cascades have been performed to examine how threshold displacement events are effected by changes in crystal structure. Exploiting the structural proximity of the rutile, anatase and brookite polymorphs of $\mathrm{TiO}_{2}$, a quantitative examination of defect production has been carried out including detailed defect analysis and the determination of values of the threshold displacement energy $\left(E_{d}\right)$. Across all polymorphs comparable values of $E_{d}$ are reported for oxygen at around $20 \mathrm{eV}$, with the value for $\mathrm{Ti}$ in rutile $(73 \pm 2 \mathrm{eV})$ significantly higher than that in brookite $(34 \pm 1 \mathrm{eV})$ and anatase $(39 \pm 1 \mathrm{eV})$. Quantifying defect formation probability as a function of Primary Knock-on Atom (PKA) energy, simulations in rutile indicate a consistent reduction in defect formation at energies higher than $E_{d}$ relative to anatase and brookite. Defect cluster analysis reveals a significant proportion of di-Frenkel pairs in anatase at Ti PKA energies around $E_{d}$. These clusters, which are stabilised by the localisation of two Frenkel pairs, are associated with a recombination barrier of approximately $0.19 \mathrm{eV}$. As such, annihilation is likely under typical experimental conditions which suggests an expected increase in the measured $\mathrm{Ti}$ value of $E_{d}$. Identical $\mathrm{O}$ defect populations produced at the threshold by the $\mathrm{O}$ PKA in both rutile and anatase explain the comparable values of $E_{d}$. At higher
\end{abstract}


O PKA energies, the commencement of defect production on both sublattices in anatase is observed in contrast to the confinement of defects to the $\mathrm{O}$ sublattice in rutile. The overall trends reported are consistent with in-situ irradiation experiments and thermal spike simulations, suggesting the contrasting radiation response of the polymorphs of $\mathrm{TiO}_{2}$ is apparent during the initial stages of defect production.

Keywords: radiation damage, computer modelling and simulation, microstructure, oxides

PACS: 61.72.Cc;61.82.Ms;61.80.Az;31.15.xv

\section{1. Introduction}

2 The threshold displacement energy, $E_{d}$, is a fundamental quantity that is cen3 tral to determining the radiation tolerance of a material. Defined as the energy 4 required to initiate defect production in a material, it can be used to relate the 5 kinetic energy of the incident primary knock-on atom (PKA) to the number of 6 resulting atomic displacements. In addition to acting as an indicator for radiation 7 tolerance, $E_{d}$ is explicitly used in various analytical models of radiation damage 8 from early models of Kinchin-Pease $[1,2]$ to models based on the binary collision 9 approximation, such as SRIM (The Stopping and Range of Ions in Matter)[3].

10 These models are used in a wide variety of applications providing estimates of 11 defect production and ion implantation depths. Significantly, for most materi12 als these estimates are poor at low incident energies where lattice effects have a 13 considerable impact on defect production.

14 In almost all situations, $E_{d}$ is considered to be simply a function of the chem15 ical composition; indeed, in SRIM calculations this is precisely the information

\footnotetext{
* Corresponding author. Address: Curtin University, GPO Box U1987, Perth, Western Australia. Tel.: +61892663780.

Email address: Marc.Robinson@curtin.edu.au (M. Robinson)

Preprint submitted to Elsevier

March 31, 2014
} 
entered into the user interface. However, for materials with multiple polymorphs, diamond and graphite being prime examples, this approximation can be far from valid. An ideal test candidate to investigate the structural dependence of $E_{d}$ is $\mathrm{TiO}_{2}$, specifically the low pressure polymorphs; rutile, anatase and brookite. The polymorphs provide a valuable opportunity to understand how subtle changes in crystal structure effect defect formation which underpins the radiation tolerance of a material. In addition, there is significant interest regarding each polymorph in their own right, due to the wide-ranging applicability of titania which includes the use of rutile as a nuclear waste form constituent.

Despite its importance, ascertaining a precise value of $E_{d}$ is difficult, particular via experiment in which the detection of the onset of defect formation is hard to accomplish. In contrast, using computer simulation to model low energy displacement cascades has been successful in determining values of $E_{d}$ for a range of materials $[4,5,6]$.

The present study is directly motivated by differences in irradiation response observed by Lumpkin et al. [7] for the low pressure polymorphs of $\mathrm{TiO}_{2}$. A combination of in-situ TEM experiments and molecular dynamics simulations illustrated the contrasting behaviour of the polymorphs and established an ordering based upon radiation tolerance. Anatase was reported to be the most susceptible to amorphisation, particularly in comparison to rutile which maintained crystallinity at fluences over an order of magnitude greater. The tolerance of brookite, although higher than anatase was also found to amorphise at significantly lower fluences than rutile.

This investigation builds upon previous studies into the rutile polymorph of $\mathrm{TiO}_{2}$ in which a systematic, automated approach to simulating low energy cascades was used to precisely determine $E_{d}[8,9]$. By incorporating the anatase and brookite polymorphs, we examine the impact of changes in crystal structure on values of $E_{d}$ and the defects produced during threshold displacement events. 


\section{Background}

45

The versatility of titania and its applications has resulted in a significant number of investigations into the polymorphs of $\mathrm{TiO}_{2}$, in particular the low pressure phases of rutile, anatase and brookite. With regard to nuclear waste form development, studies almost solely involve rutile due to a superior radiation tolerance. One of the main driving forces for the study of rutile is its utilisation in Synroctype ceramic waste-forms [10]. Additionally, rutile has a relatively simple crystal structure when compared to other waste-form constituents such as the pyrochlore family of compounds $\left(\mathrm{A}_{2} \mathrm{~B}_{2} \mathrm{O}_{7}\right)$, which is favourable when conducting fundamental studies.

Experimental studies of threshold displacement energies in rutile are limited, particularly reports of titanium values. An experimental value for Ti was reported from the TEM studies of Buck et al. [11] at between $45 \mathrm{eV}$ and $50 \mathrm{eV}$, who also determined an oxygen value of $33 \mathrm{eV}$. More recently, the ability to monitor the optical properties of anion vacancies in techniques such as TimeResolved Cathodoluminescence Spectroscopy (TRCS) has enabled more accurate oxygen values of $E_{d}$. Smith et al. [12] employed TRCS to determine $E_{d}$ in rutile, reporting a value of $34 \mathrm{eV}$.

Computational investigations utilising Molecular Dynamics (MD) have also been adopted to determined values of $E_{d}$ for rutile. Of these Richardson [13] was the first, stating values of around $50 \mathrm{eV}$ for $\mathrm{Ti}$ and values as low as $10 \mathrm{eV}$ for O. More accurate MD simulations were carried out by Thomas et al. [14], who introduced the notion of defect formation probability and emphasised the future requirement for good statistics. Values of $E_{d}$ were given in terms of the energy required to achieve a $10 \%$ defect formation probability, resulting in a value of $40 \mathrm{eV}$ for oxygen and $105 \mathrm{eV}$ for titanium. In the precursors to the present work, extensive simulations into rutile were carried out that allowed precise extraction 
71 of values of $E_{d}$ and also quantified the impact of changes in temperature and time 72 scale $[8,9]$. Values of $18 \mathrm{eV}$ and $73 \mathrm{eV}$ were determined for oxygen and titanium 73 respectively. Importantly, defect formation probability was highly sensitive to 74 temperature, even on the $10 \mathrm{ps}$ time scale of the simulations. This was a result 75 of activated Frenkel pair recombination mechanisms on the oxygen sublattice, 76 culminating in a shift in the oxygen value of $E_{d}$ from $18 \mathrm{eV}$ at $300 \mathrm{~K}$ to $53 \mathrm{eV}$ 77 at $750 \mathrm{~K}$.

In contrast to the investigations involving rutile for nuclear applications, notably fewer studies have been carried out involving anatase or brookite. This is somewhat expected due the relative susceptibility of these polymorphs in contrast to ruile. Uberuaga et al. [15] compared point defect migration behaviour

82 in the presence of grain boundaries in anatase to rutile, reporting differences in 83 damage accumulation at the boundary. At low temperature, a build up of both 84 titanium and oxygen interstitials is expected in rutile whilst in anatase defect 85 recombination is enhanced on the oxygen sub-lattice as both oxygen vacancies 86 and interstitials migrate to the boundary. Of the studies into radiation damage 87 in anatase and brookite, there are no reported values of $E_{d}$.

As a major motivation for this work, Lumpkin et al. [7] used a combination of experiment and atomistic modelling to highlight significant differences in the radiation tolerance of the rutile, anatase and brookite polymorphs of $\mathrm{TiO}_{2}$. Insitu irradiation experiments demonstrated the relative susceptibility of anatase and brookite when compared to rutile. Molecular dynamics studies in the form of thermal spike simulations fully supported this conclusion, and was investigated further in a study by Marks et al. [16]. These works demonstrated the ability of the empirical simulation approach to capture the differences in radiation response ${ }_{96}$ between the polymorphs of $\mathrm{TiO}_{2}$. Following on from this, further simulations were

the differences in tolerance between the polymorphs [17]. Indeed, the application 
of negative pressure to the rutile and brookite polymorphs to conform the three polymorphs to the same density resulted in remarkably similar radiation response. The present study aims to determine if the differences observed from the thermal spike simulations and high energy collision cascades are also apparent during threshold displacement events.

\section{Methodology}

The simulations presented in this work were carried out using a methodology developed previously $[8,9]$. which utilises the DL_POLY_4 molecular dynamics code [18]. This involved a systematic approach to both the execution and analysis of low energy cascade simulations. Calculation of quantities such as defect formation probability (DFP) as a function of PKA energy allowed precise extraction of the threshold displacement energy, $E_{d}$. Simulations employed the Buckingham pair potential parameterised by Matsui and Akoagi (MA) [19] to model the equilibrium atomic interactions. This model uses fixed partial charges of $\mathrm{q}_{\mathrm{Ti}}=+2.196|\mathrm{e}|$ and $\mathrm{qO}_{\mathrm{O}}=-1.098|\mathrm{e}|$ and includes dispersion terms for all pair interactions. To capture the nuclei-nuclei interactions at small atomic separation, the Ziegler-Biersack-Littmark (ZBL) pair potential [20] is employed. In the intermediate region, the MA and ZBL potentials are joined using an exponential function ensuring a smooth transition through to second derivatives. For potential parameters and further details regarding the potential models the reader is directed to Ref [8].

All simulations were carried out at $300 \mathrm{~K}$ for a maximum of $10 \mathrm{ps}$ and PKA energies ranged from 5 to $200 \mathrm{eV}$ at increments of $5 \mathrm{eV}$. PKA directions of initial velocity are determined by a uniform spherical sampling. Combining 100 PKA directions with 10 lattices equilibrated for varying lengths of time gives an excellent statistical ensemble of initial cascade conditions.

To determine the effect of crystal structure on values of $E_{d}$, previous results 
from simulations into rutile are combined with new sets of simulations into anatase and brookite. After preliminary testing, system sizes of 4800 atoms for anatase and 6144 atoms for brookite were found to be sufficient to contain the low energy displacement cascades. Due to the two unique oxygen sites in brookite, denoted $\mathrm{O}_{\mathrm{I}}(\mathrm{x}=0.0157, \mathrm{y}=0.1483$, and $\mathrm{z}=0.1830)$ and $\mathrm{O}_{\mathrm{II}}(0.2353$, $\mathrm{y}=0.1198$, and $\mathrm{z}=0.5301)$, the total number of simulations for this polymorph increased to 120,000 in contrast to the 80,0000 for anatase and rutile. Where appropriate, results for the two oxygen PKAs in brookite are averaged to give a single oxygen value.

In addition to calculations of quantities such as defect formation probability, the degree of statistical sampling allows for thorough defect analysis. To carry out defect cluster analysis, the residual damage was decomposed into domains using a recursive neighbour search algorithm where each constituent defect (vacancy or interstitial) was treated as a point in $3 \mathrm{D}$ space. To determine neighbouring defects a cutoff of $3 \AA$ was used. Currently, defect clusters containing more than four constituent defects are labelled as large defect clusters and no further analysis concerning such complexes is carried out. In addition to carrying out the defect cluster analysis on the complete dataset, the significant number of simulations performed at each PKA energy enabled analysis of specific energy ranges.

In the examination of defect transitions and recombination, energy barriers are calculated using the Climbing Image Nudged Elasic Band (CNEB) method $[21,22,23]$. Barriers are related to time-scales using the Arrhenius equation and a fixed attempt frequency of $10^{13} \mathrm{~s}^{-1}$.

\section{Results}

\subsection{Displacement probability}

The large number of simulations performed for each polymorph allows probing of the probabilistic nature of defect formation at low PKA energies. To gain an 
immediate understanding of the radiation response of the polymorphs, the probability of causing a displacement was calculated as a function of PKA energy and is shown in Fig. 1. This probability includes all instances in which an atom no longer resides in its original site, regardless of stable defect formation. A marked contrast in the response of the polymorphs is evident, particularly between rutile and anatase. For the Ti PKA this difference is greatest at around $80 \mathrm{eV}$, with displacements twice as likely in anatase than rutile. The most significant result from Fig. 1 is a distinct antase-brookite-rutile ordering with regards to displacement susceptibility which is consistent across the complete PKA energy range. This is directly inline with the previous experimental [7] and computational investigations $[16,17]$ and gives an initial indication that differences in radiation response between the polymorphs is present during low energy displacement events.

In the traditional definition of $E_{d}$, there is no distinction between a defect and a displacement. A permanent displacement in which an atom does not return to its original site is assumed to create a defect, with lattice effects neglected Although this assumption holds true when the system is maintained at $0 \mathrm{~K}$ and the displacement can be confidently defined as permanent, in reality the number of displacements and defects are seldom the same. Although it is possible to define $E_{d}$ using the displacement probability shown in Fig. 1, we chose to use defect formation probability as outlined in the next section to allow comparisons with experimentally derived values. This is due to the majority of experimental techniques deriving the threshold from the point at which detectable defects are formed, commonly vacancies. In this sense, $E_{d}$ should be defined as defect formation threshold. It is clear that in some materials there will be negligible differences between the threshold for causing a displacement and the threshold for defect formation, in which case the traditional definition of $E_{d}$ is suitable. 

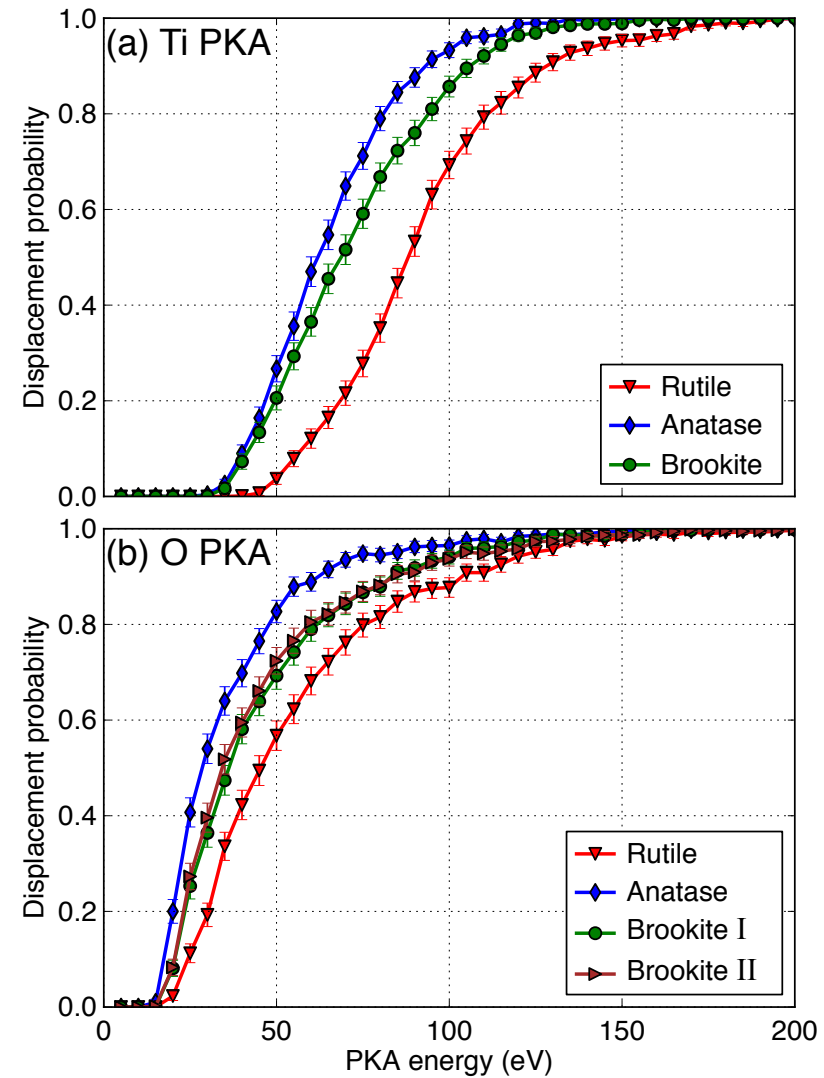

Figure 1: The probability of atomic displacement in each polymorph for the (a) Ti PKA and (b) O PKA. Error bars represent a 95\% confidence interval using the standard error in the mean. Rutile data is taken from previous simulations $[8,9]$. 


\subsection{Defect formation probability and threshold displacement energies}

To examine the response of a lattice to a displacement event an important quantity to determine is the defect formation probability (DFP) and its dependency on PKA energy. In contrast to the previously described displacement probability, DFP concerns only the formation of a vacancy-interstitial (Frenkel) pair defect which does not recombine during the 10 ps simulation. Fig. 2 shows DFP as a function of PKA energy for each polymorph and immediately indicates distinct differences between the response of rutile relative to anatase and brookite. There is a significant decrease in the propensity for defect formation in rutile, which quantitatively results in a DFP that is around $20 \%$ lower for rutile at the end of the PKA energy range.

Fig. 2 indicates negligible differences in DFP for anatase and brookite, which is in contrast to the the strong trends reported by previous thermal spike simulations and experimental observations $[7,16,17]$. This may relate to the disparity in the deposited energies between the current low energy threshold displacements and the much higher energy radiation events central to the precursory studies. In the present simulations, the local crystallinity is maintained as the cascade develops. This allows for atomic replacements and there are distinct pathways for defect recombination. However, in multiple, high energy collisions the concerted displacement of atoms renders the local environment considerably damaged. It is the recovery mechanisms during these events that may differ between anatase and brookite and as such give rise to the observed differences in irradiation response.

As introduced previously for rutile, $[8,9]$ a simple function can be used to fit DFP as a function of PKA energy:

$$
\operatorname{DFP}(E)=\left\{\begin{aligned}
0 & \text { if } E \leq E_{d} \\
\frac{1}{\beta}\left[E^{\alpha}-\left(E_{d}\right)^{\alpha}\right] & \text { if } E>E_{d}
\end{aligned}\right.
$$

o3 where $\alpha$ and $\beta$ are fitting parameters and $E$ is the energy of the PKA. The 


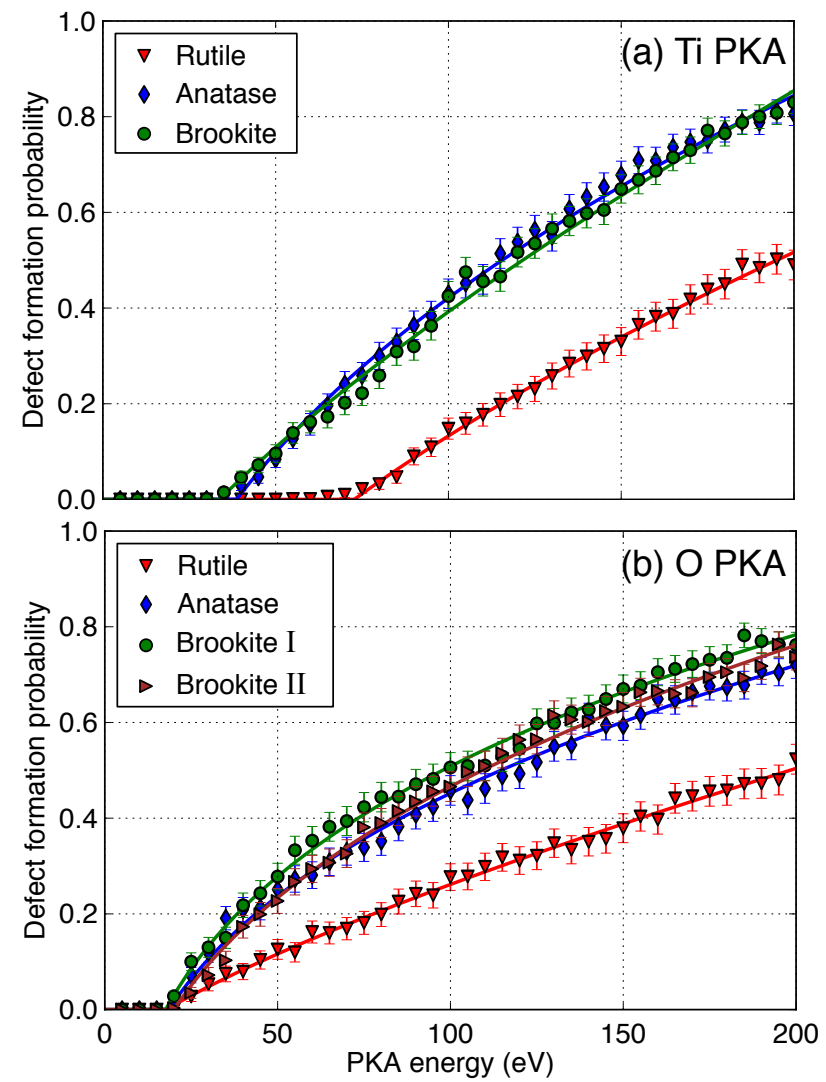

Figure 2: The probability of defect formation in each polymorph for the (a) Ti PKA and (b) O PKA. The solid lines represent the fits to Eq. 1 and error bars indicate a $95 \%$ confidence interval using the standard error in the mean. Rutile data is taken from previous simulations. [8, 9] 
piecewise form of this function explicitly includes $E_{d}$ allowing direct extraction from the fitting procedure. The final fits are represented by solid lines in Fig. 2 and the corresponding values of $E_{d}$ are shown in Table 1 . In line with previous work $[8,9]$ and Thomas et al, $[14] E_{50}$ is also reported in Table 1 which represents energies required to produce a 50\% DFP and can be readily extracted from Eq. 1.

\begin{tabular}{c||cc|ccc}
\hline \multicolumn{1}{c||}{} & \multicolumn{2}{c|}{$\mathrm{Ti}$} & \multicolumn{3}{c}{$\mathrm{O}$} \\
\cline { 2 - 6 } & $E_{d}(\mathrm{eV})$ & $E_{50}(\mathrm{eV})$ & & $E_{d}(\mathrm{eV})$ & $E_{50}(\mathrm{eV})$ \\
\hline \hline Rutile & $73 \pm 2$ & $195 \pm 2$ & & $18 \pm 3$ & $198 \pm 4$ \\
Anatase & $39 \pm 1$ & $116 \pm 2$ & & $19 \pm 1$ & $115 \pm 2$ \\
& & & $\mathrm{O}_{\mathrm{I}}$ & $18 \pm 1$ & $98 \pm 2$ \\
Brookite & $34 \pm 1$ & $121 \pm 2$ & $\mathrm{O}_{\mathrm{II}}$ & $23 \pm 1$ & $107 \pm 2$ \\
& & & &
\end{tabular}

Table 1: Values of $E_{d}$ and $E_{50}(50 \%$ DFP) for Ti and $\mathrm{O}$ for each polymorph after $10 \mathrm{ps.}$ Values are determined using the fit shown in Eq. 1. Errors represent a 95\% confidence interval calculated using the standard error in each quantity.

The values of $E_{d}$ reported in Table 1 indicate all values of $E_{d}$ for oxygen are comparable at around $20 \mathrm{eV}$. This value is lower than that reported experimentally [12] but as discussed in our previous work into rutile, is due to thermally activated recombination processes on the oxygen sub-lattice that occur within experimental time scales [9]. At higher O PKA energies, DFP is found to be lower in rutile by a similar proportion to that observed for the Ti PKA resulting in a lower value of $E_{50}$. It is possible that this behaviour relates to additional recombination processes specific to the oxygen sublattice in rutile.

In contrast to the similar values of $E_{d}$ reported for the $\mathrm{O}$ PKA across all polymorphs, a significantly higher value for the Ti PKA is found for rutile. At $73 \pm 2 \mathrm{eV}$, this is around twice that of anatase and brookite which have similar values of $34 \pm 2$ and $39 \pm 2 \mathrm{eV}$ respectively. As the Ti PKA energy increases, DFP is consistently around $25 \%$ lower for rutile up to the end of the PKA energy range which results in the lower value of $E_{50}$.

It is important to note that as reported in our previous study into rutile, DFP 
is greatly affected by temperature [9]. This was especially true for the O PKA which resulted in an increase in the value of $E_{d}$ to $50 \mathrm{eV}$ at $750 \mathrm{~K}$. Therefore any differences observed between each polymorph may be enhanced or reduced by changes in temperature. For instance, the lower values of $E_{d}$ for the Ti PKA for anatase and brookite may be shifted by an increase in temperature. This is dependent on the recombination barriers associated with the defects produced at the threshold.

The determination of DFP and displacement probability (Fig. 1) as a function of PKA energy allows us to quantify the differences in defect production between the polymorphs. As an example, a collision from a Ti PKA in anatase at an energy of $125 \mathrm{eV}$ results in a displacement probability of around $100 \%$ with a $60 \%$ DFP. For rutile however, a similar value of displacement probability of $90 \%$ is obtained, whereas the DFP is much lower at approximately $25 \%$. This gives an indication that under the same conditions, the recovery of defects is expected to be much greater in rutile.

\subsection{Ti PKA Defect analysis}

Due to the significant amount of data, further analysis will consider the rutile and anatase polymorphs only as these give the greatest contrast not only in radiation response but in properties such as density and the connectivity of the $\mathrm{TiO}_{6}$ polyhedra. In addition, no distinct differences in DFP or values $E_{d}$ are indicated between brookite and anatase and therefore it is sensible to isolate only one of these polymorphs.

\subsubsection{Defect proportions}

The extensive approach to determining $E_{d}$ enables quantitive analysis of the early stages of defect production. This allows us to determine the primary causes for the variation in values of $E_{d}$, particularly for the disparity observed for the Ti PKA. Figure 3 shows the division of defects by atomic specie for rutile and 
anatase as a function of Ti PKA energy. Significantly, at energies around the threshold values there is a marked disparity in the proportions of defects created. For rutile, there is a marginal preference for the creation of $\mathrm{O}$ defects at the threshold and as PKA energy increases $\mathrm{O}$ defects make up around $55 \%$ of the defect population. Contrasting behaviour is observed at energies around the threshold in anatase, with predominantly Ti defects created. At the threshold itself, around $90 \%$ of the defects produced in anatase are Ti defects. As PKA energy increases the proportion of Ti defects decreases, reaching a similar value to that found for rutile.

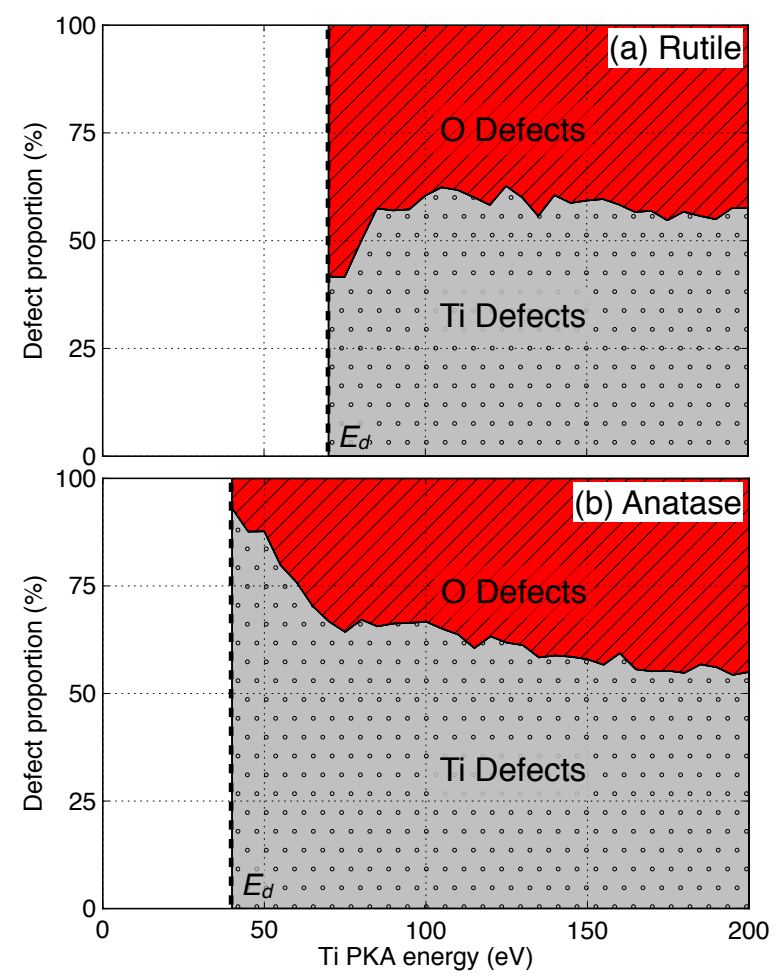

Figure 3: Average proportions of Ti and $\mathrm{O}$ defects created from the Ti PKA as a function of PKA energy for the rutile and anatase polymorphs. 
is that the Ti PKA is simply displaced to become a defect. To clarify this, the probability that the PKA forms a residual interstitial or its site a residual vacancy was calculated. At energies around the threshold this probability was found to be around $90 \%$ in anatase, whereas in rutile this was around $65 \%$. This corresponds well to the increase in the proportions of Ti defects shown in Fig. 3.

\subsubsection{Threshold directions}

To determine which directions are responsible for the defect creation at the threshold, DFP was calculated for each PKA direction. As this involves only 10 simulations per direction there is a significant reduction in statistical sampling and removes the possibility to fit DFP as a function of PKA energy. Therefore to determine the threshold energy observed for each direction, a new value was defined $E_{d}^{*}$ which represents the lowest energy at which DFP is not zero for a particular direction. This is similar to the traditional approach of calculating $E_{d}$ in which PKA energy is incremented until a single instance of defect formation is observed.

The distribution of the directional threshold energies, $E_{d}^{*}$ for Ti are shown in Fig. 4 for rutile and anatase. For both polymorphs, the values of $E_{d}^{*}$ follow an approximate normal distribution, with anatase showing a minor positive skew. A gradual threshold is present in rutile with small sets of directions producing defects at discrete energy intervals between 60 and $80 \mathrm{eV}$. In contrast, a more discrete threshold for anatase is observed with a significant number of directions producing defects around the threshold of 35 to $45 \mathrm{eV}$.

To determine if there was any relationship between the large number of directions with $E_{d}^{*}$ close to $E_{d}$ in anatase, each direction that produced a defect below $45 \mathrm{eV}$ was overlaid onto the initial PKA position. However, no significant correlation between these directions and the anatase crystal structure was apparent. We attribute this to the sampling of lattices at finite temperature which reduces 
any anisotropy that would be present in $0 \mathrm{~K}$ simulations.

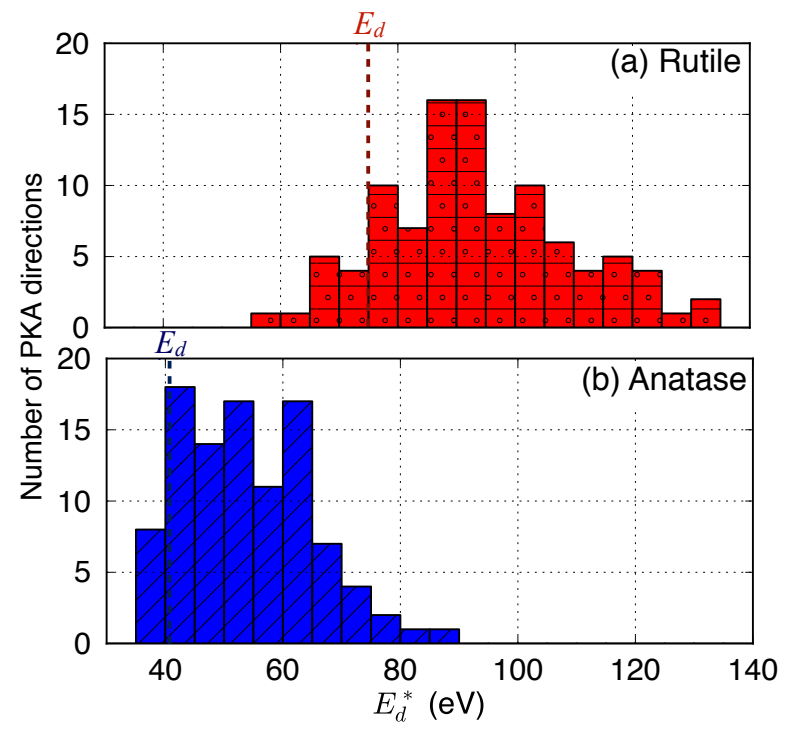

Figure 4: Ti directional threshold energies $\left(E_{d}^{*}\right)$ for each of the one hundred PKA directions simulated for (a) rutile and (b) anatase. The dashed line represents the displacement threshold energy $\left(E_{d}\right)$ extrapolated using Eq. 1 to fit DFP as in Fig.2.

\subsubsection{Defect cluster analysis}

In addition to calculating the proportions of defects in terms of the atomic specie, defect cluster analysis was also carried out. This enabled the examination of the clusters produced at energies around the threshold to determine the defects that are most readily produced. The classification of defects produced by the $\mathrm{Ti}$ PKA at energies between $E_{d}$ and $E_{d}+20 \mathrm{eV}$ is shown in Fig. 5 for both rutile and anatase. Defect clusters contributing less than $1 \%$ to the overall defect population were neglected. Fig. 5 indicates significant differences in defect production at the threshold between anatase and rutile. The most apparent disparity is the marked build up of $2 \mathrm{Ti}_{\mathrm{V}}+2 \mathrm{Ti}_{\mathrm{I}}$ clusters, herein referred to as di-Frenkel pairs (di-FPs), which in anatase contribute to over $40 \%$ of the final defect population. As these defects are considered to be made up of four constituents this explains the high 
proportion of Ti defects as seen in Fig. 3.

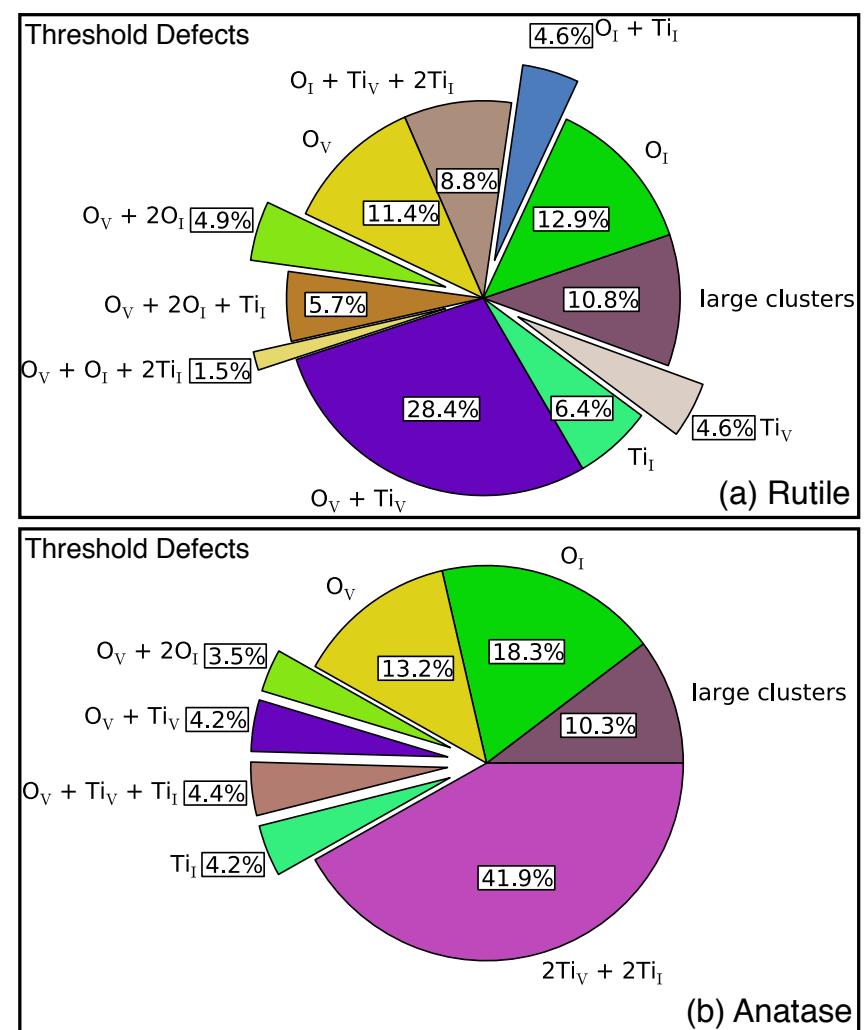

Figure 5: Defect clusters produced by the Ti PKA at energies from $E_{d}$ to $E_{d}+20 \mathrm{eV}$ in (a) rutile and (b) anatase. Clusters contributing to less than $1 \%$ of the overall defect populations are neglected. Clusters containing more than four constituent defects are labelled as large clusters.

To examine the di-FP defect further, the cluster was reconstructed in isolation in an anatase lattice. The lattice was then optimized at $0 \mathrm{~K}$ and the structure of the di-FP inspected. A diagram of the resultant cluster is provided in Fig. 6, along with the corresponding anatase lattice structure. A notable property of the di-FP is the close proximity of the constituent vacancies and interstitials. The cluster was found to always contain the PKA as an interstitial atom with its site remaining unoccupied. The second interstitial atom originated from a first nearest neighbour position resulting in a vacancy separation of $3 \AA$. The 
interstitial atoms are separated by $2.9 \AA$ and each resides in highly distorted $\mathrm{TiO}_{6}$ octahedra. Only four Ti-O bonds remain close to the $1.9 \AA$ length present in the anatase structure, with one bond at $2.3 \AA$ and the other at $2.5 \AA$. Typical coordination analysis using a cutoff of around $2 \AA$ would therefore highlight the two interstitial Ti atoms as four- or five-fold coordinated.

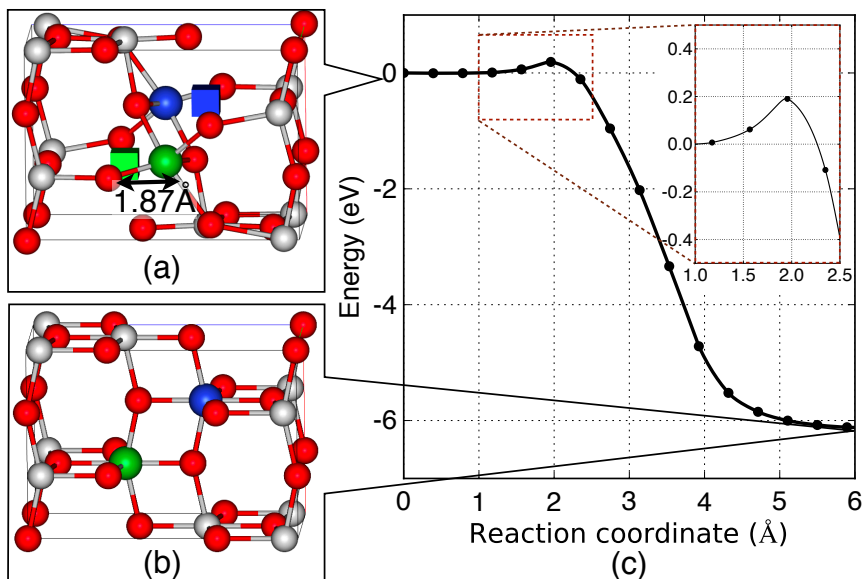

Figure 6: The di-Frenkel pair (di-FP) defect cluster and recombination energy barrier. Over $40 \%$ of the residual defects produced at energies between $E_{d}$ and $E_{d}+20 \mathrm{eV}$ from Ti PKA collisions in anatase are classified as di-FPs. (a) Defect structure consisting of two vacancies (cubes) and two displaced lattice atoms (spheres). (b) Initial anatase structure with the Ti PKA and a nearest neighbour $\mathrm{Ti}$ atom shown in blue and green respectively. (c) The recombination energy barrier calculated using the climbing image nudged elastic band method $[21,22,23]$. The reaction coordinate indicates the total displacement of all atoms during the transition between the di-FP and the perfect anatase lattice.

Significantly, the di-FP cluster was found to be stable with a formation energy of $6.1 \mathrm{eV}$ and recombination did not occur during the relaxation process. Moreover, optimisation of a single $\mathrm{Ti}_{\mathrm{V}}+\mathrm{Ti}_{\mathrm{I}}$ defect in the same configuration (a Frenkel pair) did result in recombination suggesting stability is only possible due to the localisation of the two Frenkel pairs. To determine the high temperature stability of the di-FP or equivalently the expected lifetime of cluster, the energy barrier for recombination was determined. The barrier, as shown in Fig. 6 (c) was found to be $0.19 \mathrm{eV}$ suggesting that at $300 \mathrm{~K}$ and assuming Arrhenius behaviour with a 
typical attempt frequency $\left(10^{13} \mathrm{~s}^{-1}\right)$, recombination would occur on the nanosecond time scale. This suggests that at higher temperatures or on experimental time scales, the recombination of such defects may increase the measured value of $E_{d}$ for Ti. A similar sensitivity was reported previously for the value of $E_{d}$ for $\mathrm{O}$ in rutile [9].

Aside from the differences regarding the build up of di-FPs, there are some similarities between anatase and rutile to note from Fig. 5. For instance, there are comparable proportions of lone interstitials produced in anatase and rutile on both the $\mathrm{O}$ and $\mathrm{Ti}$ sublattices. In addition, a similar proportion of $\mathrm{O}$ vacancies and $\mathrm{O}$ split-interstitials $\left(\mathrm{O}_{V}+2 \mathrm{O}_{\mathrm{I}}\right)$ are produced. Previous work involving rutile associated these lone $\mathrm{O}$ defects with low recombination barriers such that any increases in temperature shifted the observed value of $E_{d}[9]$. Combining this with the low barriers found for the recombination of the Ti di-FP defects again suggests the Ti value of $E_{d}$ in anatase is likely to increase at enhanced temperatures.

In addition to the analysis of defects clusters created at Ti PKA energies close to $E_{d}$, classification was also carried out at PKA energies in the range of 150-200 eV. The resultant defects populations for rutile and anatase are shown in Fig. 7. The main result from this analysis is the large proportion of mixed di-vacancy clusters in both polymorphs. A build-up of these defects was indicated for rutile at the threshold (Fig. 5). The proximity of these defects with O mono-vacancies could lead to the formation of Shottky defects $\left(2 \mathrm{O}_{\mathrm{V}}+\mathrm{Ti}_{\mathrm{V}}\right)$, particularly if the barrier for $\mathrm{O}$ mono-vacancy migration is lowered by the presence of the di-vacancy. Fig. 7 does indicate a degree of Schottky defect formation with around a $2-5 \%$ proportion.

Comparing the defect populations in anatase at the two energy ranges indicates a distinct reduction in the proportion of di-FPs at higher PKA energies. A simple reason for this is increased disorder caused by the higher energy impacts which reduce the likelihood of forming the complex in isolation. This is supported by 
the correlated increase in large defect clusters which may involve multiple di-FPs or a di-FP local to other defects.
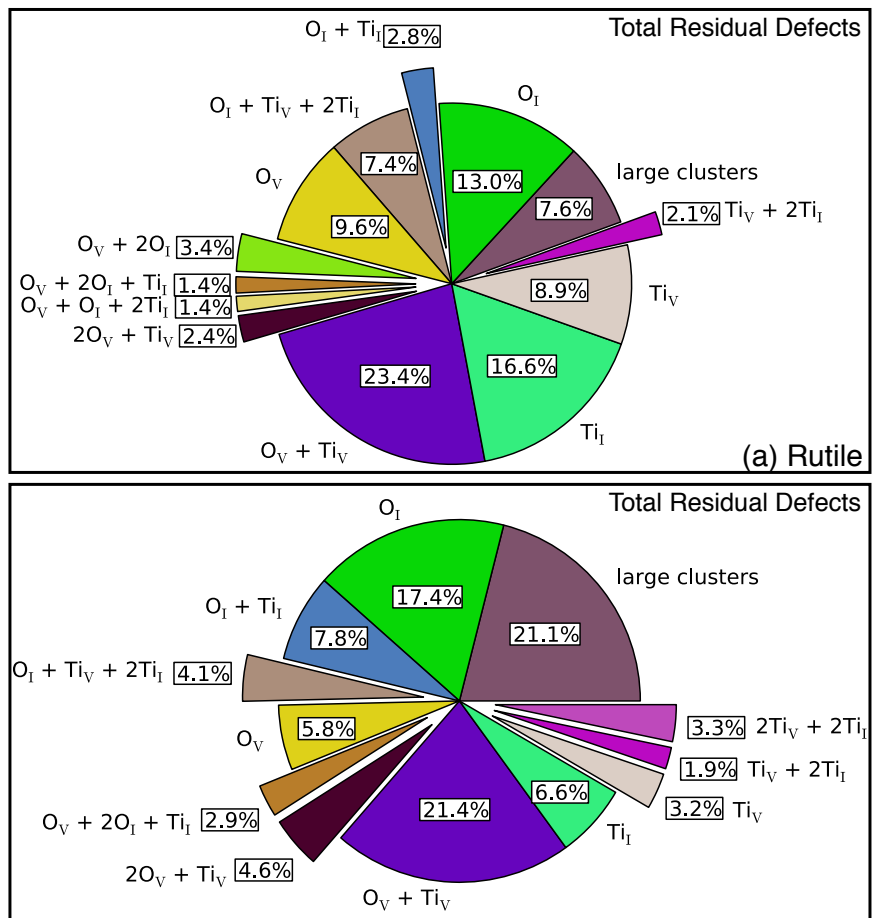

(b) Anatase

Figure 7: Defect clusters produced by the Ti PKA over the complete energy range in (a) rutile and (b) anatase. Clusters contributing to less than $1 \%$ of the overall defect populations are neglected. Clusters containing more than four constituent defects are labelled as large clusters.

\subsection{O PKA Defect analysis}

Although there we no marked differences in the values of $E_{d}$ determined for the O PKA (Table 1) at higher PKA energies a clear reduction DFP was evident for the rutile relative to the other polymorphs. To examine the cause of the changes in defect production between the threshold and higher energies, cluster analysis was again carried out. The resultant populations for both energy ranges are shown in Fig. 8 for rutile and anatase.

Compounding the similarities inferred by the similar values of $E_{d}$, defect cluster 
analysis carried out at the threshold between $E_{d}$ and $E_{d}+10 \mathrm{eV}$ generated almost identical results for both rutile and anatase. For this reason the defect proportions are shown as an average in Fig. 8 (a). In contrast to the multitude of defects produced by the Ti PKA, defects from O PKA impacts are confined to the $\mathrm{O}$ sublattice and are simple O Frenkel pairs or split-interstitials. This result was reported for rutile in our previous work [9] and the recombination of these $\mathrm{O}$ defects at higher temperatures increased the value of $E_{d}$. The similarities in values of $E_{d}$ and the residual defects produced suggest that this may also be the case for anatase.

At PKA energies between 150 and $200 \mathrm{eV}$, although there are still significant proportions of simple $\mathrm{O}$ defects in both polymorph, differences in defect production begin to emerge. For rutile, defects remain confined to the $\mathrm{O}$ sublattice and the proportions of vacancies, interstitials and split-interstitials remain unchanged from the threshold values as shown in Fig. 8 (b). For anatase however, Fig. 8 (c) indicates a build-up of defects on both sublattices with between 7 and $13 \%$ of the residual clusters containing Ti defects. The availability of additional defect structures provides an explanation for the higher probability of defect formation as O PKA energy increases. Notably, the production of additional defects in anatase includes a $3.1 \%$ build up of Ti di-FPs, suggesting that the defect commonly produced from Ti PKA displacements also forms for higher energy O PKA displacements. This is sensible given the secondary collision of the O PKA with a neighbouring Ti atom would cause a subset of defects as found for the Ti PKA.

\section{Discussions \& Conclusions}

A systematic computational study of low energy displacement events has been carried out into the low pressure polymorphs of $\mathrm{TiO}_{2}$. Combining precursory results involving rutile with the current study of anatase and brookite has allowed us to investigate how changes in crystal structure impact the initial stages of 

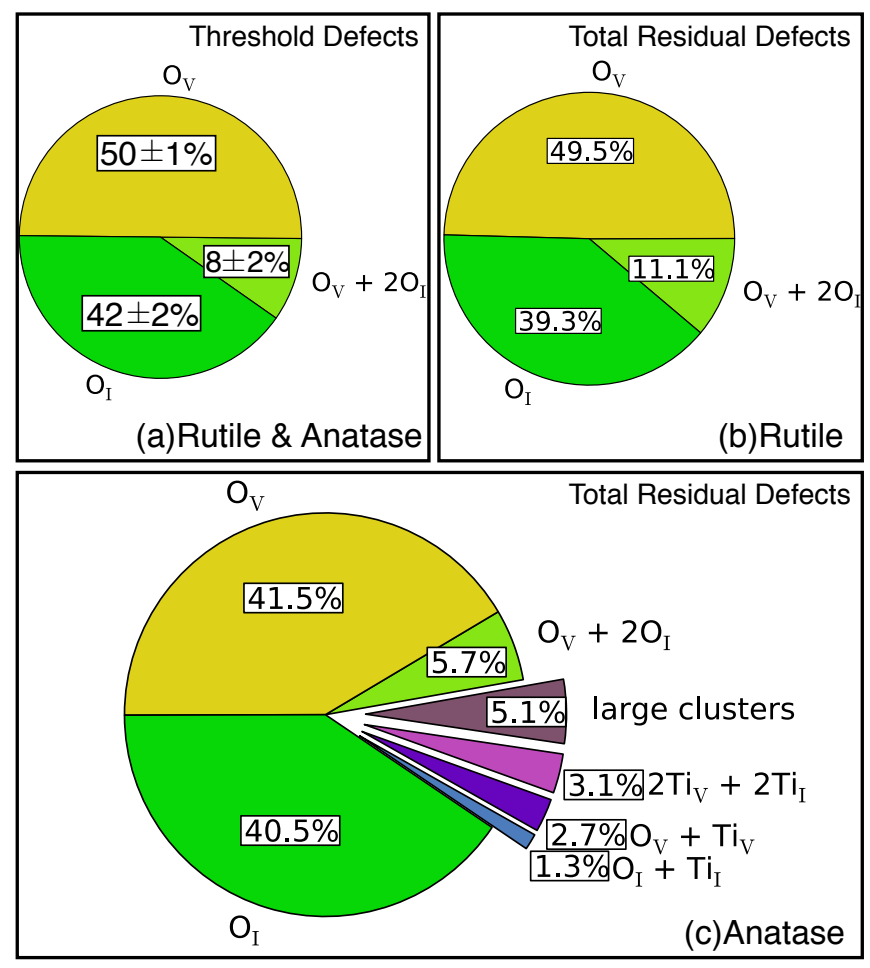

Figure 8: Defect clusters produced by the O PKA. (a) Averaged proportions of defects produced by both anatase and rutile at PKA energies around each respective threshold. (b) Total defect proportions for rutile. (c) Total defect proportions for anatase. Clusters contributing to less than $1 \%$ of the overall defect populations are neglected. Clusters containing more than four constituent defects are labelled as large clusters.

defect creation. The extensive approach adopted enables examination of the probabilistic nature of defect formation and the precise extraction of quantities such as the threshold displacement energy $E_{d}$.

Values of $E_{d}$ for titanium indicated a significant dependence on polymorph, specifically when comparing the value for rutile, $73 \mathrm{eV}$ to the similar values determined for anatase and brookite, 39 and $34 \mathrm{eV}$ respectively. Calculations of the elemental defect proportions indicated a distinct difference at energies around $E_{d}$, with the Ti defect population in anatase significantly larger than the oxygen defects. Investigating this further through defect cluster analysis highlighted the 
accumulation of a Ti defect complex in anatase which consisted of two vacancies and two interstitials. Labelled a di-Frenkel pair, stability is found to be induced by the localisation of the constituent Frenkel pairs, which if created in isolation spontaneously recombine. The energy barrier associated with the recombination of the di-Frenkel pair was calculated to be $0.19 \mathrm{eV}$ which suggests annihilation is likely on experimental time scales. A similar result was found previously for oxygen defects in rutile, which at elevated temperatures recombined and lead to an increase in the calculated value of $E_{d}[9]$.

Oxygen values of $E_{d}$ were found to be consistent across all polymorphs at around $20 \mathrm{eV}$, including the two inequivalent oxygen sites in the brookite structure. It is important to note that although values of $E_{d}$ for oxygen are comparable this is not a suitable indicator for the susceptibility to defect formation. At energies immediately higher than $E_{d}$, the probability of defect formation was significantly lower in rutile relative to the other polymorphs. This was quantified by defining $\mathrm{E}_{50}$, the energy required for a $50 \%$ probability of defect formation, and resulted in an value for rutile almost double that in anatase. This may have implications for the interpretation of previous and future studies involving $E_{d}$ in which a direct correlation between radiation tolerance and values of $E_{d}$ is suggested.

The overall contrasting behaviour of rutile to the other polymorphs, especially anatase, is in line with our previous experimental and computational studies[16, 7, 17]. In particular, the calculated displacement probabilities correlate directly with the ordering of radiation tolerance reported, with rutile the most tolerant, followed by brookite and finally anatase. As the previous studies involved significantly higher energies than the current simulations, displacement probability is a better quantity for comparison as apposed to defect formation probability. This is due to the contrasting degree of localised disorder, which is minimal in the current simulations and allows mechanisms of recombination 
either during the ballistic phase or through thermal activation.

A by-product of the comprehensive sets of simulations and complimentary defect analysis, is a representative catalogue of defects for each polymorph. With the ongoing developments in techniques that simulate the long time scale dynamics of defects, $[24,25,26,27]$ such a database is a necessity. Therefore, studies adopting the approach used in this work may not only serve to examine the dynamics of defect formation but to also provide representative starting configurations for simulations of defect migration and damage evolution.

\section{Acknowledgements}

The work was supported by computing resources at the iVEC facility located at Murdoch University.

\section{References}

[1] G. H. Kinchin and R. S. Pease, Reports Prog. Phys. 18, 1 (1955).

[2] M. J. Norgett, M. T. Robinson, and I. M. Torrens, Nucl. Eng. Des. 33, 50 (1975).

[3] J. F. Ziegler, M. D. Ziegler, and J. P. Biersack, Nucl. Instrum. Meth. B 268, 1818 (2010).

[4] K. Nordlund, J. Wallenius, and L. Malerba, Nucl. Instrum. Meth. B 246, 322 (2006).

[5] R. Smith, D. Bacorisen, B. P. Uberuaga, K. E. Sickafus, J. A. Ball, and R. W. Grimes, J. Phys.: Condens. Matter 17, 875 (2005).

6] B. S. Thomas, N. A. Marks, and B. D. Begg, Nucl. Instrum. Meth. B 254, 211 (2007).

[7] G. R. Lumpkin, K. L. Smith, M. G. Blackford, B. S. Thomas, K. R. Whittle, N. A. Marks, and J. Z. Zaluzec, Phys. Rev. B 77, 1 (2008).

[8] M. Robinson, N. A. Marks, K. R. Whittle, and G. R. Lumpkin, Phys. Rev. B 85, 104105 (2012).

[9] M. Robinson, N. Marks, and G. Lumpkin, Phys. Rev. B 86, 1 (2012).

[10] A. E. Ringwood, S. E. Kesson, N. G. Ware, W. Hibberson, and A. Major, Nature 278, 219 (1979).

[11] E. C. Buck, Radiat. Eff. Defects Solids 133, 141 (1995).

[12] K. L. Smith and N. J. Zaluzec, J. Nucl. Mater. 336, 261 (2005).

[13] D. D. Richardson, Radiat. Eff. Defects Solids 79, 75 (1983).

[14] B. Thomas, N. Marks, L. Corrales, and R. Devanathan, Nucl. Instrum. Meth. B 239, 191 (2005).

[15] B. P. Uberuaga and X.-M. Bai, J. Phys.: Condens. Matter 23, 435004 (2011).

[16] N. A. Marks, B. S. Thomas, K. L. Smith, and G. R. Lumpkin, Nucl. Instrum. Meth. B 266, 2665 (2008).

[17] M. J. Qin, E. Y. Kuo, K. R. Whittle, S. C. Middleburgh, M. Robinson, N. A. Marks, and G. R. Lumpkin, J. Phys.: Condens. Matter 25, 355402 (2013).

[18] I. T. Todorov, W. Smith, K. Trachenko, and M. T. Dove, J. Mater. Chem. 16, 1911 (2006).

[19] M. Matsui, M. Akaogi, Mol. Simul. 6, 4 (1991). 
[20] J. F. Ziegler, J. P. Biersack, and U. Littmark, The stopping and range of ions in matter (Pergamon, New York, 1985).

[21] G. Henkelman, B. P. Uberuaga, and H. Jnsson, J. Chem. Phys. 113, 9901 (2000).

[22] G. Henkelman and H. Jnsson, J. Chem. Phys. 113, 9978 (2000).

[23] B. Johansson, G. Mills, and K. W. Jacobsen, in Classical and Quantum Dynamics in Condensed Phase Simulations, edited by B. Berne, G. Ciccotti, and D. Coker (World Scientific, Singapore, 1998), p. 385

[24] C. Domain, C. Becquart, and L. Malerba, J. Nucl. Mater. 335, 121 (2004).

[25] L. Xu and G. Henkelman, J. Chem. Phys. 129, 114104 (2008).

[26] H. Xu, Y. N. Osetsky, and R. E. Stoller, J. Phys.: Condens. Matter 24, 375402 (2012).

[27] L. K. Béland, P. Brommer, F. El-Mellouhi, J.-F. Joly, and N. Mousseau, Phys. Rev. E 84, 046704 (2011). 